\title{
Comparison of Nasopharyngeal Swabs with Salivary Samples for Viral Nucleic Acid Detection from Clinical and Radiologically Confirmed Cases of Sars-Cov-2 in a Tertiary Care Centre, Thanjavur
}

\author{
M. Sathiyalakshmi, Eunice Swarna Jacob*, Sivagamasundari, Sangeetha, \\ S. Maruthuthurai and Hari Prasanth
}

Department of Microbiology, Govt Thanjavur Medical college, Thanjavur - 613 004, India

*Corresponding author

A B S T R A C T

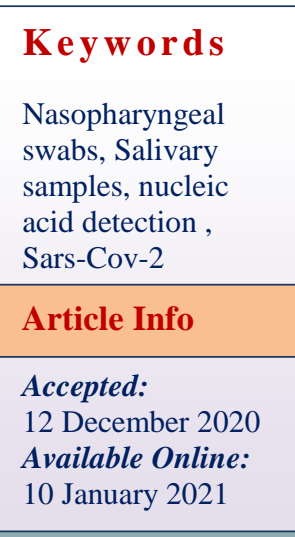

Keywords

Nasopharyngeal swabs, Salivary samples, nucleic acid detection Sars-Cov-2

Article Info

Accepted:

12 December 2020

10 January 2021 with the increasing number of Pandemic Corona virus disease 2019 (COVID-19) cases, there is a need for a quick and easy method to obtain a non-invasive sample for the detection of this novel corona virus (severe acute respiratory syndrome corona virus 2; SARS-CoV-2). This study aimed to investigate the potential use of salivary samples as a non-invasive tool for the diagnosis of COVID-19. Study Period: October 2020 to November 2020.Type of Study: Prospective study. Place of study: Covid Care Centre, Tertiary Hospital Thanjavur. Specimens: A total of 60 samples of Saliva and nasopharyngeal swabs from SARI patients admitted in Covid care center Thanjavur during the outbreak of COVID-19. Real-time polymerase chain reaction (RT-PCR) was performed, and the results of the two specimens were compared. Sixty participants were enrolled in this study which included 33 males and 27 females. The median age was 32 years (IQR: 25-51 years). A positive test by either sample route (saliva/NPS) tested by qRT-PCR was considered as true positive and considered as reference standard. The 9 participants who were NPS tested negative for COVID-19, also tested negative with saliva (True negatives). Out of 44 positive cases, 42(70\%) were accurately diagnosed as SARS Co-V-2 positive with saliva and $44(73 \%)$ with NPS. With saliva samples the sensitivity was $84 \%$ specificity was $90 \%$, positive predictive value was $97.67 \%$, negative predictive value was $52.94 \%$ and accuracy was $85 \%$. Whereas with NPS the sensitivity was $88 \%$, specificity was $80 \%$, positive predictive value was $95.65 \%$, negative predictive value was $57.14 \%$ and accuracy was $86.67 \%$. The Cycle threshold values of both the sampling methods were comparable. Saliva was also found to be equally consistent. $70 \%$ of the participants found saliva as the preferred method of sampling.

\section{Introduction}

The World Health Organization (WHO) reported cases of pneumonia of unknown aetiology in Wuhan city, China in December
2019. The disease was identified to be caused by a novel corona virus which was later named as Severe Acute Respiratory Syndrome Corona Virus-2 (SARS-CoV-2) and the disease was named Corona Virus 
Disease 2019 (COVID-19). It has been declared as a pandemic by the WHO (1). SARS-CoV-2 employs the host cell angiotensin-converting enzyme 2 as the main host receptor for cellular entry (2). Previous experimental studies showed a higher level of angiotensin-converting enzyme 2 expressions in salivary glands compared with that in the lungs (3) and the epithelial cells lining salivary gland duct were early target cells of SARS-CoV infection in rhesus macaques (4). SARS-CoV was also detected in saliva samples (5). This suggested that the salivary glands could be a potential target for SARCoV-2 infection, and hence saliva could be a potential sample for SARS-CoV-2 detection (6). Accurate and rapid diagnostic testing is a key factor to control the spread of pandemic caused by the novel corona virus (7). Real Time Reverse Transcriptase Polymerase Chain Reaction (qRT-PCR) on respiratory specimens like Oropharyngeal Swab (OPS), Nasopharyngeal Swab (NPS), bronchoalveolar lavage and tracheal aspirates, is the current gold standard test for detection of SARS CoV-2. The most commonly used sample type is NPS which has been found to be inconsistent during serial testing and has relatively poor sensitivity in early infection (7). The collection of NPS has various disadvantages like it requires close contact between the healthcare worker performing the collection and the patient. This exposes the healthcare workers to aerosols generated due to sneezing and coughing by the patient during sample collection, putting them at high risk. The other disadvantages include invasiveness of the procedure, which causes discomfort and risk of bleeding in thrombocytopenic patients.

This study compares the sensitivity and specificity of paired salivary and NPS, qRTPCR with total qRT-PCR as the reference standard for diagnosis of COVID-19 infection.

\section{Materials and Methods}

A cross-sectional study was conducted among 60 SARI patients who were admitted in Covid ward at tertiary care center, Thanjavur Medical College during the period from October 2020 - November 2020. Samples collected from 60 patients who were diagnosed with viral pneumonitis admitted in Covid care centre confirmed as COVID 19 by real time RT PCR and Plain CT thorax of those individuals(CORADS scoring ). The inclusion criteria includes Symptomatic patients with nasopharyngeal swab positive cases and Radiologically confirmed Covid positive patients. A written informed consent was taken from all participants. The patients who were on drugs affecting saliva production like anticholinergics, had xerostomia or any active oral disease or injury and mechanically ventilated or critically ill patients were excluded from the study. Patient characteristics, symptoms at presentation and risk factors were collected. The samples were collected with all aseptic precautions in accordance with the current institutional Standard Operating Procedures (SOP). NPS was collected by a trained health care professional using a flexible, flocked swab which was passed through the patient's nostril until the posterior nasopharynx was reached. It was left for 10 seconds in place to absorb secretions and removed slowly while rotating. The swab was put in $3 \mathrm{ml}$ sterile Viral Transport Medium (VTM) and sealed securely. The participant was requested not to eat, drink, smoke or chew gum for at least 30 minutes before giving saliva sample. Prior to spitting, participant was asked to relax and rub cheeks gently for 30 seconds to make saliva. The sample of 2-3 $\mathrm{ml}$ saliva (minus any bubbles that form on the top of the vial) took around 5 minutes to collect. Participants self-collected the saliva sample using the drooling technique, in a sterile vial containing $3 \mathrm{ml}$ of VTM, which was sealed and shared 
with the laboratory personnel. No special saliva collection devices were used.

This study was approved by Institutional Ethical Committee For Human Studies, Thanjavur Medical College, Thanjavur (India) (Certificate number:799/2020).

\section{Specimen processing}

The Specimens were labeled with unique laboratory numbers. Laboratory personnel who performed specimen processing and RTPCR were unaware of the names and hospital numbers of the participants. Nasopharyngeal swabs in VTM and saliva samples from the collection container were treated with lysis buffer to inactivate the SARSCoV-2. Viral RNA was extracted from $200 \mathrm{~mL}$ of the samples within 30 minutes using Helini automated nucleic acid extraction system, according to the manufacturer's instructions.

\section{RT-PCR workflow}

The detection of SARS-CoV-2 in the specimens was performed by RT-PCR amplification of the SARS-CoV-2 RDRP and $\mathrm{N}$ gene fragments, using a SARS-CoV-2 Nucleic Acid Diagnostic Kit (labgun exofast), which was approved for the detection of SARS-CoV-2 by Indian council of medical research . The lower limit of detection of the test was 100 copies /sample.

The detection of human RNase $\mathrm{P}$ gene was included in the kit as a control. RT-PCR was performed using the CFX96 Real-Time Detection System (BioRad). The result was considered positive if the cycle threshold $(\mathrm{Ct})$ values of both target genes were 10- 30 and negative when $\mathrm{Ct}$ values of both targets were less than 10 and more than 30. The turnaround time of the diagnosis was approximately 4 hours.

\section{Statistical analysis}

Data were analysed for normality and descriptive statistics were presented as a number (\%) for categorical variables and mean \pm standard deviation (SD) or median (interquartile range; IQR) for continuous variables. Chi-square or Fisher's exact test was used for categorical variables. Sensitivity, specificity, positive predictive value, negative predictive value and a $95 \%$ CI were calculated to assess diagnostic performance. All statistical analyses were performed using Graph pad prism Version 5.

\section{Results and Discussion}

60 sample pairs of nasopharyngeal swabs and saliva samples were collected. Thirty -three $(62 \%)$ individuals were men and twentyseven $(38 \%)$ individuals were women. To determine the diagnostic performance of saliva and NPS tests, the cumulative positive qRT-PCR results of both samples were used as the reference standard and the sensitivity and specificity of saliva and NPS, qRT-PCR were calculated separately. Tables 1 and 2 depict the diagnostic performance of salivary qRT-PCR and NPS qRT-PCR. The sensitivity of salivary qRT-PCR samples was $84 \%$ (95\%CI: 70.89\%e92.83), specificity was 90 $\%$ (95\%CI:55.50e99.75), positive predictive value was $97.67 \%(95 \%$ CI: $86.70 \%$ e99.63\%), negative predictive value was $52.94 \%$ (95\% CI:36.58\%e68.69\%) and accuracy was 85\%(95\%CI:73.43\%e92.90\%). Whereas the sensitivity of NPS qRT-PCR was $88 \%$ (95\%CI:75.69e95.47) specificity was $80 \%$ (95\%CI:44.39\%e97.48\%), positive predictive value was $95.65 \%$ (95\%CI: $86.38 \%$ e.98.74\%), negative predictive value was $57.14 \%$ (95\%CI:37.18\%e $75.02 \%$ ) and accuracy was $86.67 \%$ (95\%CI: $75.41 \%$ e.94.06\%). Thus, the sensitivity, negative predictive value and accuracy of nasopharyngeal swabs were higher than salivary samples; but specificity 
and Positive predictive value were higher for salivary samples. Both NPS and salivary samples were found to be equally consistent. We can say that salivary samples qRT-PCR is equally sensitive and accurate as compared to NPS. RNase P gene (Internal control) was detectable in all specimens. The median
(IQR) $\mathrm{Ct}$ values of the RDRP and $\mathrm{N}$ genes were $23(15 \mathrm{e} 30)$ and $22(14 \mathrm{e} 28)$, respectively in saliva specimens, and $24(14 \mathrm{e} 32)$ and 25 (15e30), respectively, in nasopharyngeal swabs. Ct values of the positive specimens are shown in the table 3.

Table.1 Detection of SARS Co-V-2 using saliva qRT-PCR

\begin{tabular}{|l|c|c|c|}
\hline \multicolumn{4}{|c|}{ saliva qRT-PCR } \\
\hline & Disease positive & Disease negative & total \\
\hline Test positive & 42 & 1 & 43 \\
\hline Test negative & 8 & 9 & 17 \\
\hline total & 50 & 10 & 60 \\
\hline
\end{tabular}

Table.2 Detection of SARS Co-V-2 using NPS qRT-PCR

\begin{tabular}{|l|c|c|c|}
\hline \multicolumn{4}{|c|}{ NPS qRT-PCR } \\
\hline Test positive & Disease positive & Disease negative & total \\
\hline Test negative & 44 & 1 & 45 \\
\hline total & 6 & 9 & 15 \\
\hline
\end{tabular}

Table.3 Median ct value N gene and RDRP gene

\begin{tabular}{|l|c|c|}
\hline Type of gene & $\begin{array}{c}\text { Ct value Saliva by } \\
\text { qRT-PCR }\end{array}$ & $\begin{array}{c}\text { Ct value NPS by } \\
\text { qRT-PCR }\end{array}$ \\
\hline & median & median \\
\hline N gene & $22(14 \mathrm{e} 28)$ & $25(15 \mathrm{e} 30)$ \\
\hline RDRP gene & $23(15 \mathrm{e} 30)$ & $24(14 \mathrm{e} 32)$ \\
\hline
\end{tabular}

Rapid screening and timely diagnosis of SARS Cov-2 during the pandemic is quite essential in breaking the chain of transmission and intensity resulting in reduced morbidity and mortality in clinical health care settings. Apart from the level of sensitivity and specificity of laboratory techniques, selecting the appropriate sites to collect samples is very important. Selection of proper sampling method should be based on the tissue affinity of targeted virus, cost-effectiveness of method and also safety of patients and clinicians. In this study we classified the current evidence regarding the reliability of saliva as a diagnostic specimen in COVID-19 patients. Most of the studies included in this review, reported that there is no statistically significant difference between nasopharyngeal or sputum specimens and saliva samples regarding viral load. These studies suggested saliva as a non-invasive specimen type for the diagnosis and viral load monitoring of SARSCoV-2.From recent studies, SARS-CoV-2 was detected from posterior oropharyngeal saliva samples, with a notable high viral load at the disease 
presentation. In this study, an early morning a saliva sample was self-generated by the individual, without a need for coughing up. This non-invasive procedure of saliva collection might be less aerosol-generating and might reduce the risk of infection for health-care workers working in the clinic. Although one might argue that the collected specimens were possibly mixed between saliva and sputum, the chance of patient's coughing up phlegm is rather low, as a recent study showed that dry cough was the most common symptom presented in approximately $80 \%$ of patients at the onset of the illness. Although testing of saliva samples might provide an advantage as a simple procedure, a comparison of diagnostic studies between saliva sample and confirmed-case bronchoalveolar lavage fluid or convalescence serum titre has not been available. A recent study that detected the virus from multiple sites showed a lower test positivity rate from the nasal swab (63\%) compared with bronchoalveolar lavage fluid (93\%). Therefore, a lower detection rate of SARS-CoV-2 from saliva, compared with bronchoalveolar lavage fluid, among individuals with severe disease might be possible. Of interest, two specimens had detectable SARS-CoV-2 from saliva samples, but not from nasopharyngeal and throat swabs.

Previous studies also reported a high overall agreement between saliva and nasopharyngeal aspirate specimens when tested by an automated multiplex molecular assay approved for point-of-care testing. Based on these studies, the method of collection of saliva and collection device types are critical issues in the way of using saliva as diagnostic specimen. In this regard there are three main types of human saliva (whole saliva, parotid gland and minor gland) and the method of collection of each type varies accordingly. When the aim of sampling is detecting the respiratory viruses with molecular assays, collecting the whole saliva from the suspected patients is useful. In this regard the patients should be instructed to expectorate saliva into a sterile container. The volume of saliva should be ranged between $2-3 \mathrm{ml}$. Then $1 \mathrm{ml}$ of distilled water added to the container and vortex at $12000 \mathrm{rpm}$ at 5 minutes.

The next procedures will be conducted based on instructions of related RT-PCR technique in the microbiology laboratory. The low concordance rate of saliva with nasopharyngeal specimens reported in the research of Chen et al., might be explained by the differences in the method of obtaining the samples. This study reported the detection rate of SARS-CoV-2 in pure saliva fluid secreted from the opening of salivary gland canals. However in other studies patients were asked to cough out saliva from their throat into sterile containers, and hence the saliva samples were mainly sputum from the lower respiratory tract. Thus for increasing the sensitivity of salivary tests in the way of diagnosing the suspected COVID-19 patients, the instructions should clearly explain the correct procedure to the individuals. The use of saliva samples for diagnosis of SARSCoV2 has many advantages in clinical practice. First, collecting saliva is a noninvasive procedure and rather than nasal or throat swabs avoids patient discomfort. The second advantage of using saliva as specimen is related to possibility of collecting samples outside the hospitals. This sampling method doesn't require the intervention of healthcare personnel and the suspected patients can provide it by themselves. Therefore this method can decrease the risk of nosocomial SARS-CoV-2 transmission. Furthermore, because there is no necessary for presence of trained healthcare workers for collecting saliva specimen, the waiting time for suspected patients will be reduced. This is crucial in busy clinical settings where a large 
number of individuals require screening. The results of viral culture in one of the included studies showed that saliva collected from COVID-19 patients, may contain live viruses which may allow transmission of virus from person to person (9). These finding reinforce the use of barrier-protection equipment as a control measure, for all healthcare workers in the clinic/hospital settings during the epidemic period of COVID-19. It should be mentioned that this study has several limitations. This study didn't evaluate other factors such as severity of disease or disease progression that may impact on detection rate of the virus.

Finally as all of the selected studies only included hospitalized confirmed COVID-19 patients, further studies should be performed in outpatient settings. The yield of the saliva specimen as a complementary diagnostic test for COVID-19 needs further study. As for the limitations, our study only focused on saliva testing among symptomatic individuals under investigation but the spectrum of the disease ranges from asymptomatic, through upper respiratory tract symptoms and pneumonia, to acute respiratory distress syndrome. Therefore, the performance of the saliva test for the detection of SARS-CoV-2 among asymptomatic individuals remains unknown

In conclusion, the present study proves saliva to be a viable, more sensitive, accurate, consistent and non-invasive method of sampling for diagnosing COVID-19. In resource limited setting like ours, where the cost of PPE and swabs are putting an extra burden on the health care system, we should consider alternate sample types like saliva which is also cost-effective, safer and more sensitive for detecting SARS Co-V-2 virus. We assert that saliva can be further evaluated as a valid frontline non-invasive diagnostic test for detection of COVID-19.

\section{References}

1.Gupta N, Potdar V, Praharaj I, Giri S, Sapkal G, et al., (2020) Laboratory preparedness for SARS-cov-2 testing in India: Harnessing a network of Virus Research \& Diagnostic Laboratories. Indian J Med Res 151:216- 225.

2.Azzi L, Carcano F, Gianfagna F, Grossi P, Gasperina D, et al., (2020) Saliva as a reliable tool to detect SARS-cov-2. J Infect 81:e45-e50.

3.Hoffmann M, Kleine-Weber H, Schroeder S, Kruger N, Herrler T, et al., (2020) SARS-cov-2 cell entry depends on ACE2 and TMPRSS2 and is blocked by a clinically proven protease inhibitor. Cell 18:271-280. 4. Xu J, Li Y, Gan F, Du Y, Yao Y (2020) Salivary glands: Potential reservoirs for COVID-19 asymptomatic infection. J Dent Res 99:989.

5.Wang WK, Chen SY, Liu IJ, Chen YC, Chen HL, et al., (2004) Detection of SARS associated coronavirus in throat wash and saliva in early diagnosis. Emerg Infect Dis 10:1213-1219.

6.Paso sub E, Watcharananan SP, Boonyawat $\mathrm{K}$, Janchompoo P, Wongtabtim $\mathrm{G}$, et al., (2020) Saliva sample as a noninvasive specimen for the diagnosis of coronavirus disease 2019: A cross sectional study. Clin Microbial Infect.

7.Wyllie AL, Fournier J, Casanovas-Massana A, Campbell M, Tokuyama M, et al., (2020) Saliva is more sensitive for SARS-cov-2 detection in COVID-19 patients than nasopharyngeal swabs. Medrxiv.

8.Chang MC, Hur J, Park D (2020) Interpreting the COVID-19 test results. Am J Phys Med Rehabil 99:583-585.

9.KK, Tsang OT, Chik-Yan Yip C, Chan KH, Wu TC, et al., (2020) Consistent detection of 2019 novel coronavirus in saliva. Clin Infect Dis. 
10.Vaz SN, Santana DS, Netto EM, Pedroso C, Wang WK, et al., (2020) Saliva is a reliable, non-invasive specimen for SARS-CoV-2 detection. Braz J Infect Dis. 11. Bajaj N, Granwehr BP, Hanna
EY, Chambers MS (2020) Salivary detection of SARS-CoV-2 (COVID-19) and implications for oral healthcare providers. Head Neck 42:1543-1547.

\section{How to cite this article:}

Sathiyalakshmi, M., Eunice Swarna Jacob, Sivagamasundari, Sangeetha, S. Maruthuthurai and Hari Prasanth. 2021. Comparison of Nasopharyngeal Swabs with Salivary Samples for Viral Nucleic Acid Detection from Clinical and Radiologically Confirmed Cases of Sars-Cov-2 in a Tertiary Care Centre, Thanjavur. Int.J.Curr.Microbiol.App.Sci. 10(01): 1195-1201. doi: https://doi.org/10.20546/ijcmas.2021.1001.143 